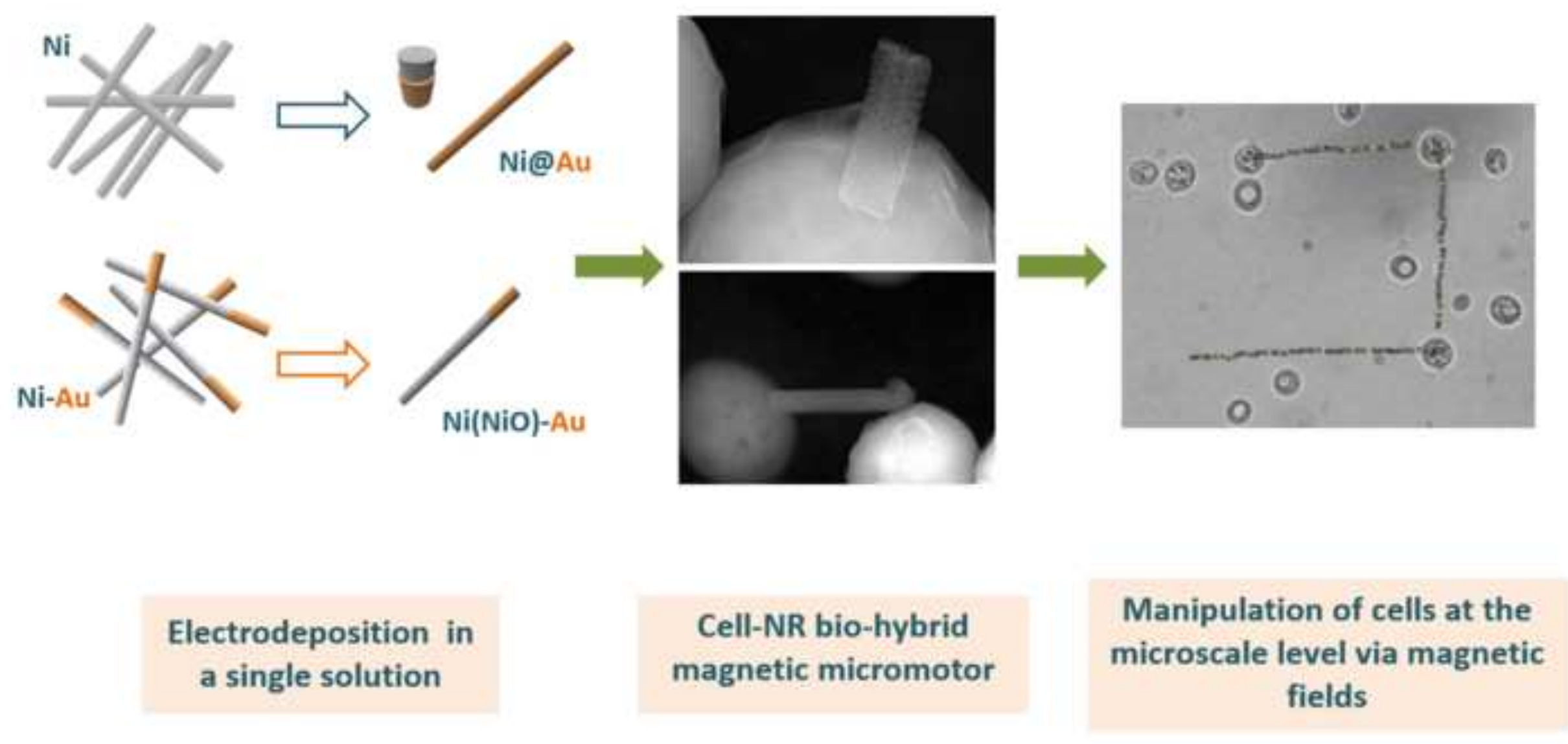

Cell-NR bio-hybrid magnetic micromotor
Manipulation of cells at the
microscale level via magnetic
Manipulation of cells at the
microscale level via magnetic fields

(n)

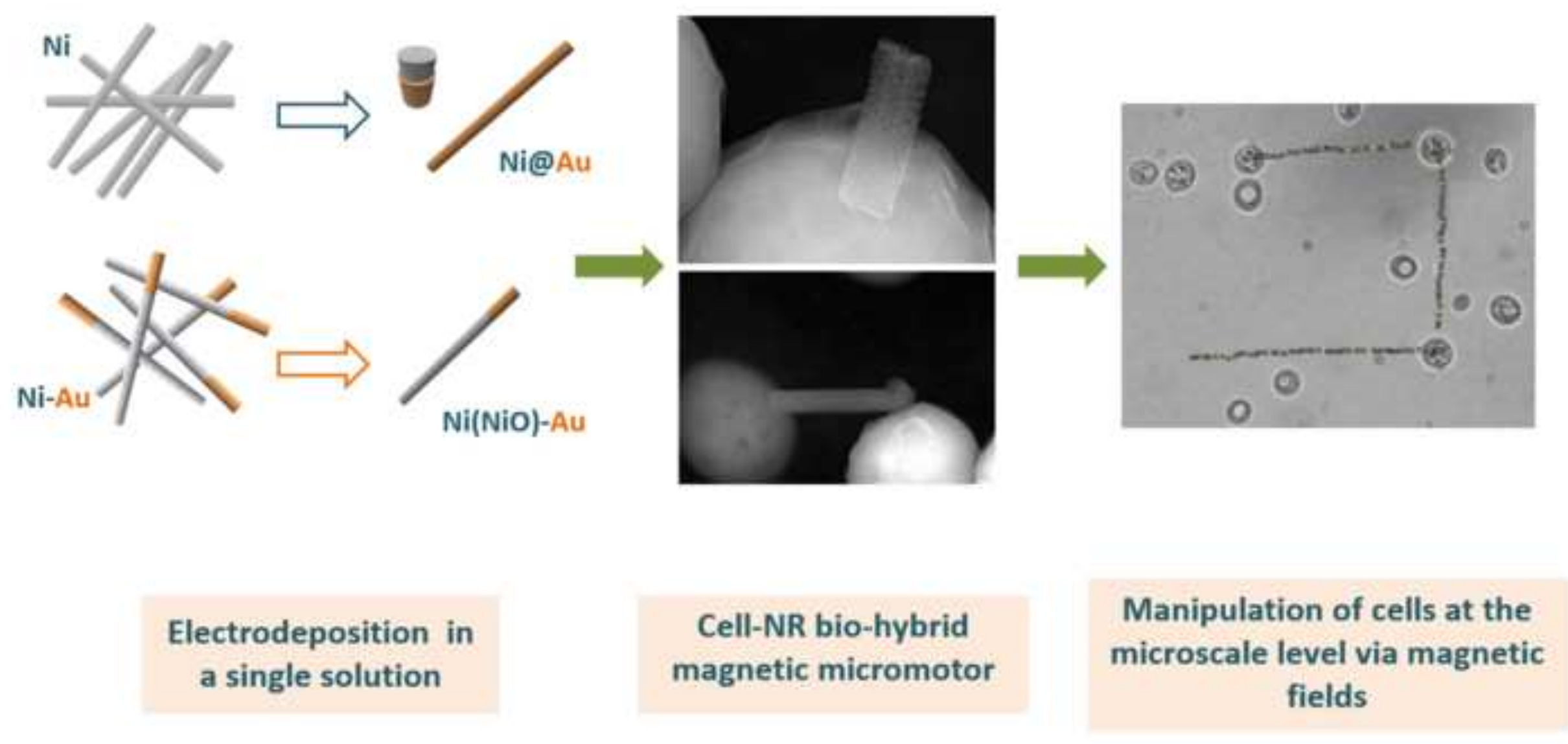

\author{
a single solution
}

Ni@Au
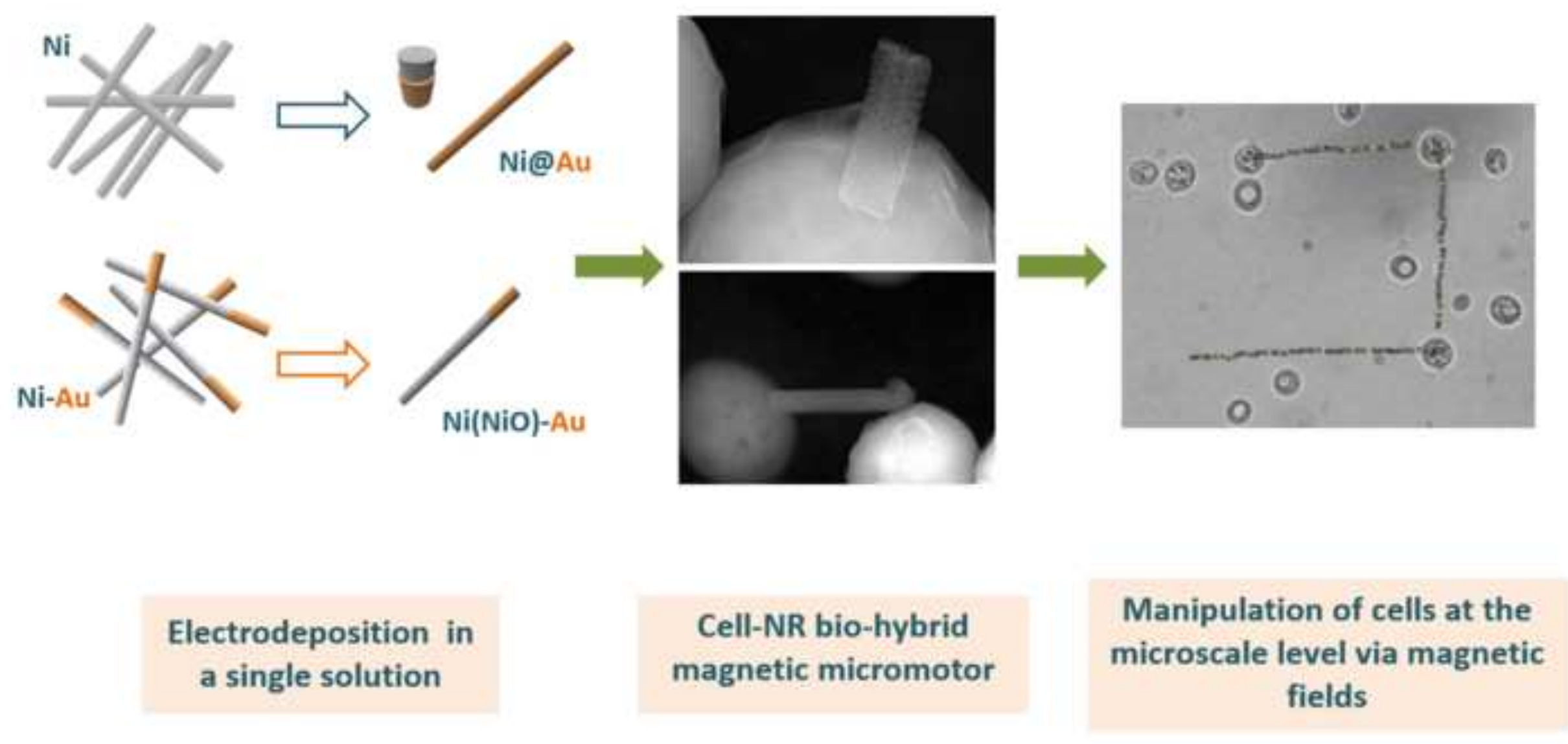
Highlights

- Electrochemical synthesis of two types of magnetic nanomotors in a single solution

- Core-shell Ni@Au and Ni@NiO-Au nanorods designed to avoid cytotoxicity

- Formation of cell-nanorod biohybrid asymmetric microstructures

- Controlled movement of yeast cells with micron-size precision

- Strategy for separation of cells and biomedical applications 


\title{
Electrochemically synthesised nanostructures for the manipulation of cells: Biohybrid micromotors
}

\author{
Albert Serră $^{1,2}$, Elisa Vallés ${ }^{1,2}$, José García-Torres $^{1,2,3, *}$ \\ ${ }^{1}$ Grup d'Electrodeposició de Capes Primes i Nanoestructures (Ge-CPN), Departament de \\ Ciència de Materials i Química Física, Universitat de Barcelona, Martí i Franquès, 1, E-08028, \\ Barcelona, Catalonia, Spain \\ ${ }^{2}$ Institute of Nanoscience and Nanotechnology (IN $\left.{ }^{2} \mathrm{UB}\right)$, Universitat de Barcelona. \\ ${ }^{3}$ Departament de Física de la Matèria Condensada, Martí i Franquès, 1, E-08028, Barcelona, \\ Catalonia, Spain.
}

*Corresponding author: jmgarcia@ecm.ub.edu

\begin{abstract}
In this communication we describe the preparation and operation of two types of $\mathrm{Ni} / \mathrm{Au}$ nanorods (NRs) as new magnetic micromotors for the transport and manipulation of single cells to target specific areas with micron-size precision. This enables huge potential environmental or biomedical applications at the cellular scale. Electrodeposition followed by chemical treatment was employed to fabricate biocompatible Ni/Au NRs in two different configurations: nonhomogeneous bi-segmented Ni@NiO-Au and homogeneous core-shell Ni@Au NRs. After incubating the NRs with yeast cells for $24 \mathrm{~h}$, they were partially taken into the cells, forming cells-NR biohybrid microstructures. We demonstrate that the asymmetric hybrid structures can be driven by external magnetic fields. The velocity and direction of the cells' motion can be controlled and tuned by modifying the field strength, frequency or direction of the rotating magnetic field. Similar hydrodynamic behaviour is observed for biohybrid microstructures containing the two types of NRs.
\end{abstract}

Keywords: electrodeposition, magnetic nanorods, micromotors, cell manipulation, biohybrid microstructures

\section{1.-Introduction}

Nano/micromotors are nano/microscale devices designed to perform specific actions after navigating to a particular place. Some of them have been used to control the manipulation of 
live cells using external stimuli (e.g. light, ultrasonic and acoustic waves, electric fields, temperature) [1-5]. Among those stimuli, magnetic actuation has garnered a great deal of attention as it offers advantages including easy manipulation, control of the swimming dynamics via the applied magnetic field, and wireless control, all without any harmful effects on the human body.

Given their potential for magnetic actuation, magnetic nanostructures have attracted increasing attention for use in biomedical applications (e.g. drug delivery, imaging, cell manipulation) [6,7]. However, locomotion of these nanostructures represents the first challenge because at the nano/microscale the low Reynolds number environment makes movement difficult as the viscous forces dominate the inertial ones. Consequently, hydrodynamics becomes time reversible and no net motion is observed [8,9]. The design of an efficient micromotor thus requires both a swimming strategy that operates under these low Reynolds number constraints, and a navigation strategy for overcoming the viscous forces. Another challenge is the possible cytotoxicity of the metallic nanostructures. For example, nickel nanowires several microns long increase in toxicity $24 \mathrm{~h}$ after incubation due to the partial dissolution of $\mathrm{Ni}$ in cells [10]. To overcome this core-shell structures can be used in magnetic manipulation to decrease toxicity, as well as achieving significant biocompatibility [11,12]. The shell acts as a barrier to the dissolution of the metallic magnetic core. Another strategy to decrease toxicity is to synthesize partially combined magnetic material with noble metals such as gold $[13,14]$.

This communication aims to use biocompatible and magnetic Ni/Au nanorods (NRs) for the control and manipulation, at the micrometric level, of individual test yeast cells after the incorporation of these magnetically anisotropic nanostructures into the cell. Specifically, two different Ni/Au configurations - homogeneous core@ shell and non-homogeneous bi-segmented - were prepared by electrodeposition followed by chemical treatment to confer biocompatibility and avoid possible nickel toxicity. Electrodeposition has been selected because it is well suited to fabrication of nanostructures with varied properties $[15,16]$. Moreover, this technique has several advantages over other methods, such as physical vapor deposition, chemical synthesis, etc. These advantages include fast deposition rates, room temperature synthesis, easy set-up and process scalability. We demonstrate here that living cells can be steered in a fluid once they have been combined with the NRs. The NRs make it possible to tune the velocity and direction near a substrate by modifying the frequency, amplitude and direction of the rotating magnetic field, as a consequence of the asymmetry in the cell-NR hybrid micromotor. The proposed strategy is very interesting as it allows the transport of cells to the required target while retaining biocompatibility because of the outer inert layers of the NRs. 


\section{2.-Experimental}

The NRs were fabricated by electrodeposition from a single bath $-0.75 \mathrm{M} \mathrm{NiCl}_{2}+5 \mathrm{mM}$ $\mathrm{HAuCl}_{4}+0.15 \mathrm{M} \mathrm{H}_{3} \mathrm{BO}_{3}$ at $\mathrm{pH}=4$ - prepared with distilled water treated with a Millipore Milli $\mathrm{Q}$ system. The electrosynthesis of mono-segmented $(\mathrm{Ni})$ and bi-segmented (Ni-Au) NRs was conducted potentiostatically using a microcomputer-controlled potentiostat/galvanostat Autolab with PGSTAT30 equipment, GPES software and a three-electrode system. The three-electrode system consisted of a polycarbonate (PC) membrane $(20 \mu \mathrm{m}$ thick, $100 \mathrm{~nm}$ nominal pore diameter, and $10^{8}-2.5 \cdot 10^{9}$ pores $\mathrm{cm}^{2}$ ) with a 100 -nm thick gold layer on one side to make the PC membrane electrically conductive, a $3 \mathrm{M} \mathrm{Ag} / \mathrm{AgCl} / \mathrm{KCl}$ electrode, and a spiral platinum wire as working, reference, and auxiliary electrodes, respectively. After synthesis of the NRs, they were released from the PC membrane and washed. Next, the Ni NRs were submerged in a solution of $20 \mathrm{mM} \mathrm{HAuCl}_{4}$ for $90 \mathrm{~s}$, leading to the formation of an $\mathrm{Au}$ shell via a galvanic displacement reaction (Ni@Au NRs). In addition, the Ni-Au NRs were submerged in a $0.5 \mathrm{M}$ $\mathrm{NaOH}$ solution for $6 \mathrm{~h}$, leading to the formation of a nickel oxide shell via nickel oxidation (Ni@NiO-Au NRs). Finally, the nanorods were incubated for $24 \mathrm{~h}$ in a petri dish containing yeast cells (Levanova, fresh yeast) dispersed in water. Sucrose (Sigma Aldrich) was used to check if the yeast cells were dead or alive.

The morphology and structure of the NRs were characterized by scanning electron microscopy and back-scattered electron detection (BSE) in a field-emission scanning electron microscope (FE-SEM, Jeol JSM-2100F). The morphology of the yeast cells and the NRs/yeast cell micromotors were also investigated by scanning electron microscopy (FE-SEM, Jeol JSM2100F) [17]. The hybrid micromotors were propelled by an external magnetic field generated using three custom-made coils oriented along three perpendicular directions. Each coil consisted of $\sim 1100$ turns of $4 \mathrm{~mm}$ diameter wire and had outer and inner diameters of 5 and $2 \mathrm{~cm}$, respectively. The rotating magnetic field was achieved by connecting two of the three coils to a waveform generator (TTi TGA1244) controlled by a current amplifier (IMG STA-800). Different frequencies $(1-60 \mathrm{~Hz})$ and magnetic field strengths $(2-6 \mathrm{mT})$ were applied to study their influence on the net movement of the nanorods. The nanorods were imaged with a $100 \times$ oil immersion objective mounted on a light microscope (Nikon). Videos for image analysis were taken at 75 fps using a charge-coupled device color camera (Basler A311F).

\section{3.-Results and discussion}

Two types of magnetic nanorods ( $\mathrm{Ni} @ \mathrm{Au}$ and $\mathrm{Ni} @ \mathrm{NiO}-\mathrm{Au}$ ), $100 \mathrm{~nm}$ in diameter and a few microns in length, were synthesized in a single bath. The cyclic voltammogram of the solution using the gold-coated PC membrane as working electrode shows a small reduction peak around $600 \mathrm{mV}$ and a second peak at $-800 \mathrm{mV}$, attributed to $\mathrm{Au}(\mathrm{III})$ and $\mathrm{Ni}(\mathrm{II})$ reduction processes, 
respectively (Figure 1(a)). On the basis of this study, the best potentials to electrodeposit pure gold $(-500 \mathrm{mV})$ and practically pure nickel $(-1000 \mathrm{mV})$ were selected in order to grow nanorods inside the channels of the membranes. Ni@Au NRs were prepared by potentiostatic deposition of $\mathrm{Ni}$ at $-1000 \mathrm{mV}$ (Figure 1(c)), followed by removal of the Ni NRs from the membrane, exhaustive cleaning and, lastly, formation of a gold shell over the Ni NRs by means of galvanic displacement in a $20 \mathrm{mM} \mathrm{HAuCl}_{4}$ solution. This protocol, previously tested for CoNi NRs [12], allowed the formation of biocompatible and stable core-shell $\mathrm{Ni@Au} \mathrm{NRs.} \mathrm{In} \mathrm{addition,}$ $\mathrm{Ni@NiO-Au} \mathrm{bi-segmented} \mathrm{NRs} \mathrm{were} \mathrm{prepared} \mathrm{by} \mathrm{applying} \mathrm{two} \mathrm{consecutive} \mathrm{potentials} \mathrm{to} \mathrm{the}$ $\mathrm{Ni}-\mathrm{Au}$ solution, the first $(-500 \mathrm{mV})$ to form the Au segment and the second $(-1000 \mathrm{mV})$ to grow the magnetic Ni segment (Figure 1(d)). The clear difference between the intensities of the two processes was due to the significant difference between the concentrations of $\mathrm{Au}$ (III) and $\mathrm{Ni}$ (II). The lengths of the two segments could be adjusted by controlling the deposition charges. To ensure the biocompatibility of these bi-segmented $\mathrm{Ni} / \mathrm{Au} \mathrm{NRs}$, following release from the membrane and washing, they were immersed in a $0.5 \mathrm{M} \mathrm{NaOH}$ solution for $6 \mathrm{~h}$ to form a complete nickel oxide layer [14]. Figure 1(b) shows a scheme for treatment of $\mathrm{Ni}$ and $\mathrm{Ni}-\mathrm{Au}$ nanorods designed to protect them and form biocompatible Ni@Au and Ni@NiO-Au NRs. 
(a)

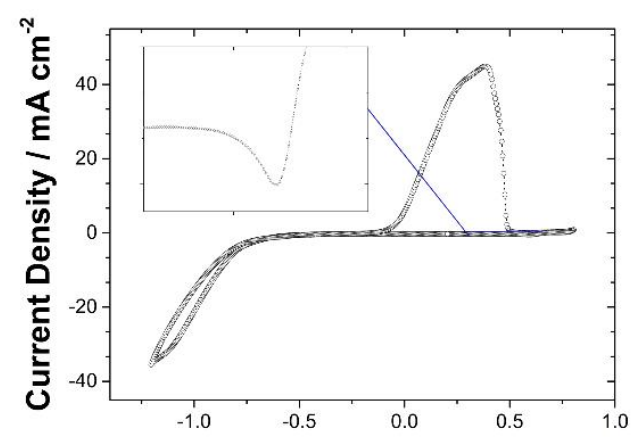

(c)

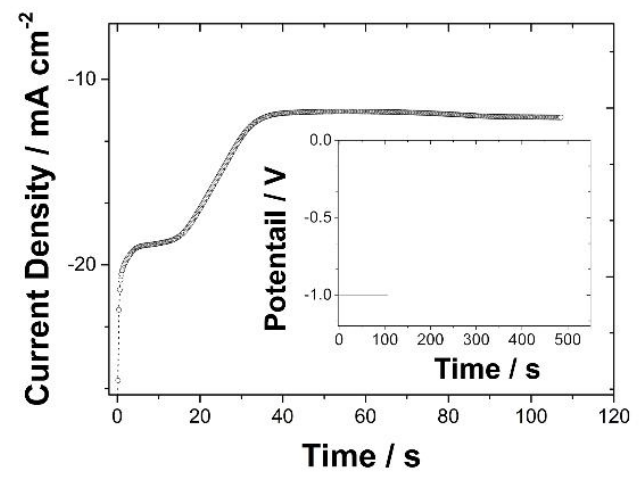

(e)

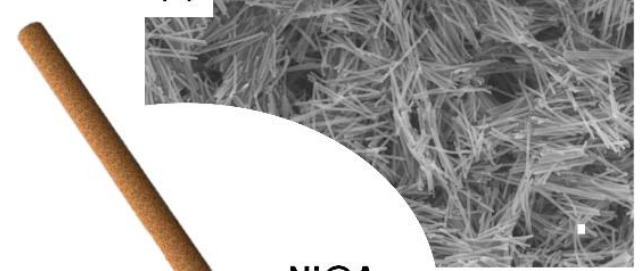

Ni@Au

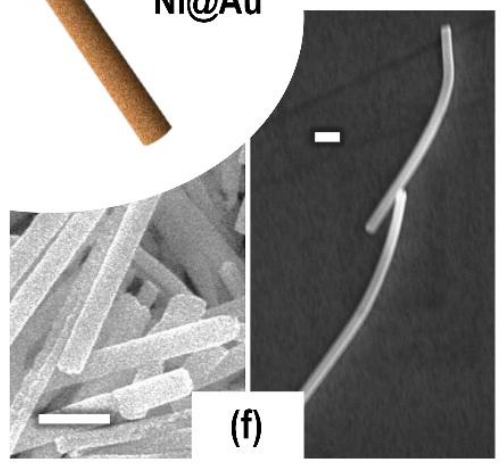

(b)
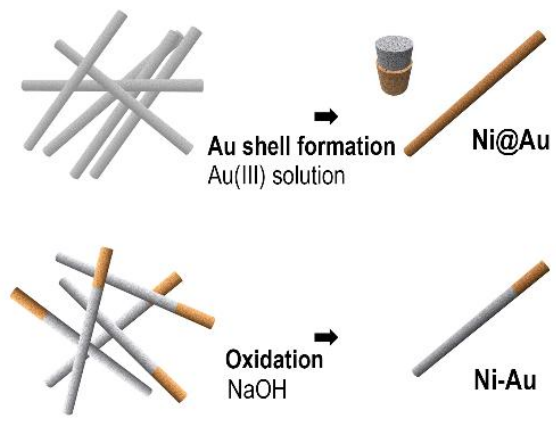

(d)

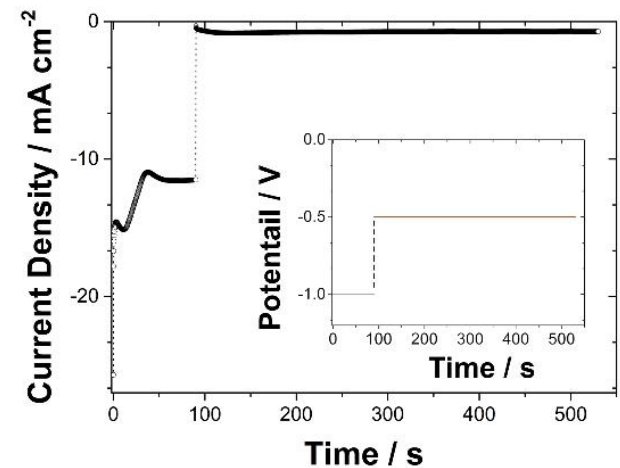

(g)



Figure 1: (a) Cyclic voltammetry of electrochemical bath at $25^{\circ} \mathrm{C}$ with a scan rate of $20 \mathrm{mV} \mathrm{s}^{-1}$ using gold-coated PC membranes as the working electrode. Inset: Enlargement of gold reduction peak. (b) Schematic representation of the surface treatments of the NRs. (c,d) Chronoamperometric curves of the electrosynthesis inside the nano-channels of the PC membranes of (c) Ni and (d) Ni-Au NRs. Inset: Applied potential during electrodeposition of (c) Ni mono-segmented and (d) Ni-Au bi-segmented NRs. Typical (e,g) FE-SEM and (f,h) BSE FE-SEM micrographs of (e,f) Ni@Au mono-segmented and (g,h) Ni@NiO-Au bi-segmented NRs (scale bar: $200 \mathrm{~nm}$ ). 
The FE-SEM images of the synthesized and treated NRs show their uniformity and good definition (Figure 1(e) for $\mathrm{Ni} @ \mathrm{Au}$ and 1(g) for $\mathrm{Ni} @ \mathrm{NiO}-\mathrm{Au}$ ), and yielded an estimated average length of 4 microns in both cases. Back-scattered (BSE) FE-SEM observation revealed the uniformity of the Ni@Au NRs (Figure 1(f)) and the presence of two clear segments in the $\mathrm{Ni} @ \mathrm{NiO}-\mathrm{Au}$ NRs.

Yeast cells were observed in Millipore water, demonstrating that they live for at least $48 \mathrm{~h}$. The cell viability was corroborated by the reaction of the cells with sucrose, which liberated $\mathrm{CO}_{2}$ gas. Live yeast cells were incubated with the two types of synthesized NRs. Figure 2 shows a comparison of the cell cultures before and after incubation with the NRs. After $24 \mathrm{~h}$, interaction between the cells and the NRs was detected, with some of the cells showing adhesion or incorporation of the NRs (Figures 2(b) and c)), to form a biohybrid cell-NR asymmetric microstructure.

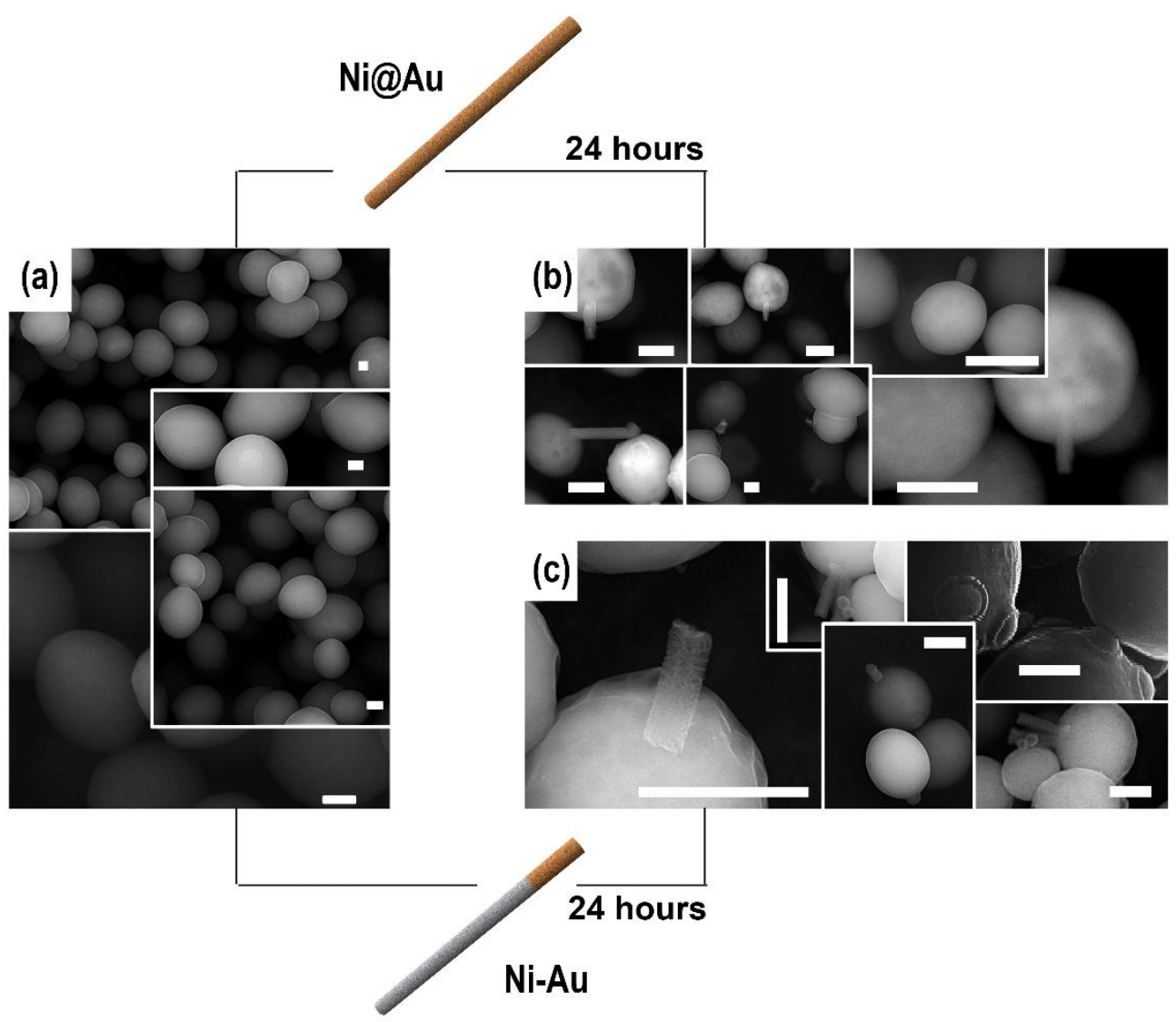

Figure 2: Typical FE-SEM images of (a) yeast cells and (b,c) biohybrid cell-NR micromotors (b) Ni@Au core@shell and (c) Ni@NiO-Au bi-segmented NRs - deposited from suspensions in ethanol (99\%) onto Si/Ti/Au plates. Scale bar: $400 \mathrm{~nm}$. 
Next, we studied the motion of yeast cell-NR hybrid structures under an external rotating magnetic field circularly polarized in a plane perpendicular to the substrate, $\vec{H}=H_{0}(0, \cos (2 \pi f t)$ - $\sin (2 \pi f t)$ ), where $H_{0}$ is the amplitude and $f$ the frequency. Biohybrid structures were propelled in different directions by changing the direction of the applied magnetic field. Figure 3a-c shows a sequence of images demonstrating how the cell-NR structure can swim in the $x$ or $y$ direction when the rotating magnetic field was in the $x z$ or $y z$ plane (Figures 3a, 3b). Moreover, the motion could be reversed by changing the direction of rotation of the magnetic field (Figure 3c) (Video S1, supporting information). The possibility of tuning directionality is a key feature for applications such as medical nano/microrobotics.

When microscopic objects are immersed in a fluid they must accommodate their motion to highly viscous environments. Under these conditions, inertia is negligible and the resulting hydrodynamic laws lead to fluid flows which are time-reversible. This implies that swimming by reciprocal motion (e.g. based purely on symmetric backward and forward body distortions, and showing one degree of freedom) is not possible at the microscale as it is at the macroscale $[8,9]$. In our biohybrid microstructure, the time reversal symmetry is broken in a rotating magnetic field. The applied field tries to align the anisotropic ferromagnetic NR - with a permanent moment $m$ oriented along its long axis - through the net torque exerted by the field. Moreover, the presence of the yeast cell at one end of the NR and the substrate generates asymmetry and therefore a difference in friction between the two ends that results in a net translational motion.

Figure $3 \mathrm{~d}$ shows the average speed as a function of the frequency for different field amplitudes and for the two different NRs. For a certain magnetic field strength, velocity increases linearly with $f$ in a synchronous regime up to a maximum. Beyond that, the hybrid microstructure enters an asynchronous regime and $v$ decreases. The critical frequency at which the dynamics change from synchronous to asynchronous depends on the magnetic field strength, as this controls the magnetic torque. Therefore, increasing $H_{0}$ raises the transition frequency and the velocity of the hybrid propeller. The same trend is obtained for both types of nanorods independently of the distribution of $\mathrm{Ni}$ and $\mathrm{Au}$ in core-shell or bi-segmented structures. Moreover, similar velocities were obtained for both kinds of nanorods, $25 \mu \mathrm{m} / \mathrm{s}$ being the highest velocity achieved. 
(a)
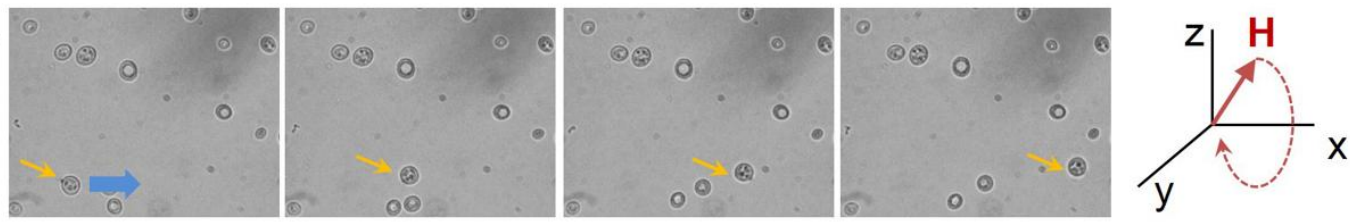

(b)
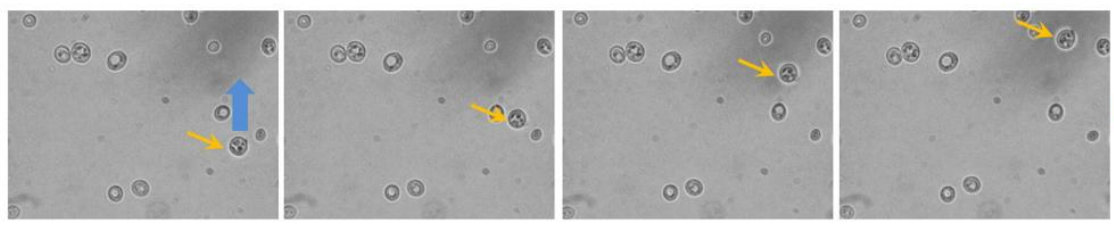

(c)
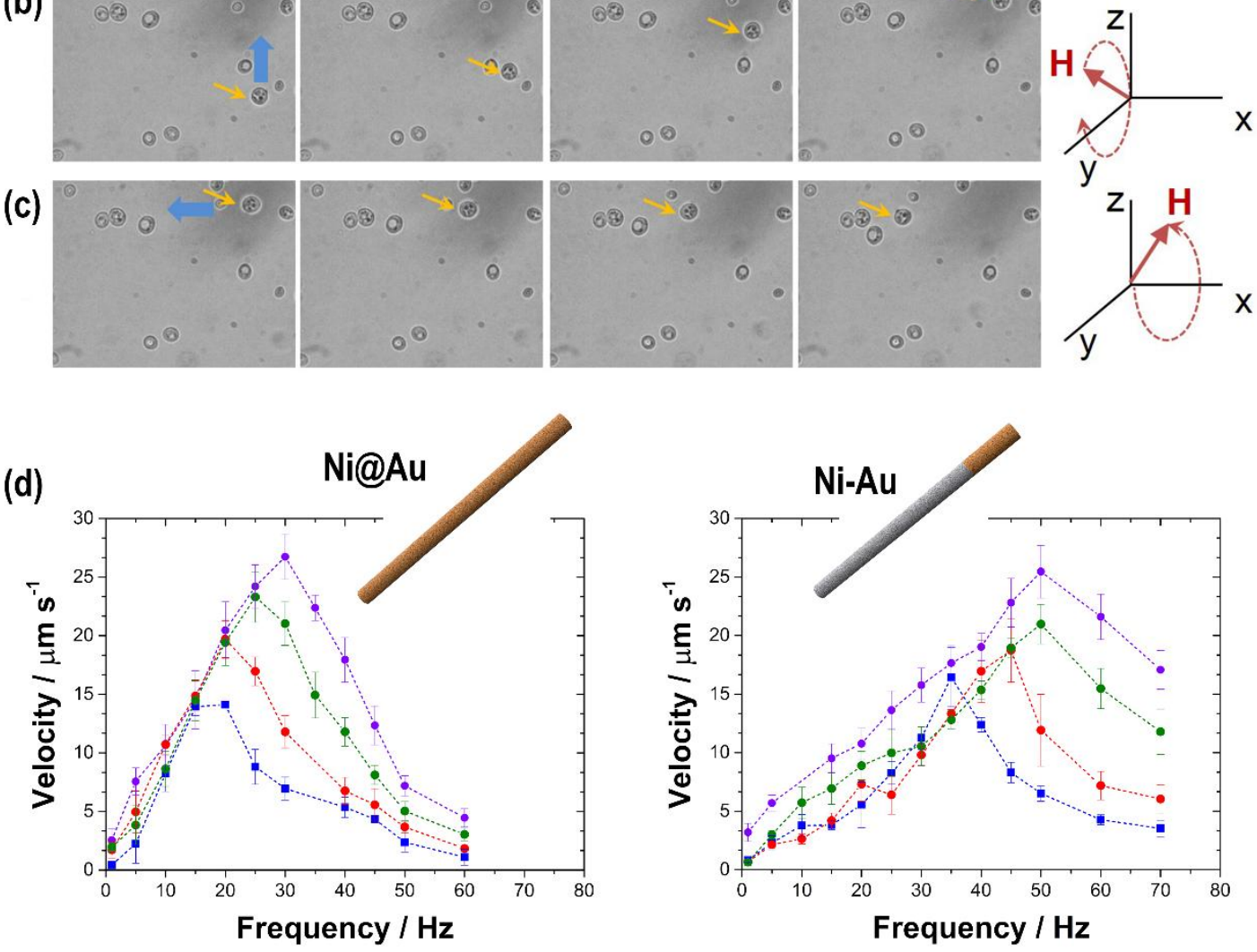

$\ldots . .5 .4 \mathrm{mT} \quad \ldots . \ldots 4.5 \mathrm{mT} \quad \ldots .3 .4 \mathrm{mT} \quad \ldots 2.5 \mathrm{mT}$

Figure 3: (a-c) Image sequences showing the motion of a yeast cell-NR biohybrid micromotor in different directions depending on the applied rotating magnetic field: (a) rotating magnetic field in the $x z$ plane, $4.5 \mathrm{mT}, 5 \mathrm{~Hz}$, (b) rotating magnetic field in the $y z$ plane, $4.5 \mathrm{mT}, 5 \mathrm{~Hz},(\mathrm{c})$ rotating magnetic field in the $x z$ plane, $4.5 \mathrm{mT}, 5 \mathrm{~Hz}$. The direction of rotation of the magnetic field is opposite to the field in (a). The orange arrow indicates the yeast-cell NR micromotor and the blue arrow the direction of motion. (d) Velocity $v s$ frequency plots for the $\mathrm{Ni@Au}$ core@shell and Ni@NiO-Au bi-segmented NRs.

\section{Conclusions}

We have successfully fabricated two types of biocompatible magnetic NRs (Ni@Au and $\mathrm{Ni@NiO-Au)} \mathrm{in} \mathrm{a} \mathrm{two-step} \mathrm{process:} \mathrm{electrodeposition} \mathrm{using} \mathrm{a} \mathrm{single} \mathrm{solution} \mathrm{followed} \mathrm{by} \mathrm{a}$ chemical treatment to confer biocompatibility. These NRs have been shown to be very 
successful as magnetically steered micromotors for the strict control and manipulation of cells at the microscale level. The cell-NR biohybrid micromotors were obtained after incorporation of the NRs into the yeast cells during incubation. The asymmetric nature of the biohybrid micromotor and the substrate allow it to be propelled magnetically in a controlled way in terms of direction and velocity. It is believed that this strategy - different types of biocompatible and magnetically-anisotropic NRs controlled via magnetic actuation - could successfully contribute to the separation or manipulation of cells at the microscopic level.

\section{Acknowledgements}

This work was supported by the EU ERDF funds and Spanish Government Grant TEC201451940-C2-2-R from Ministerio de Economía y Competitividad (MINECO). The authors thank the Centres Científics $i$ Tecnologics de la Universitat de Barcelona (CCiT-UB) for the use of their equipment.

\section{References}

[1] H. Mulvana, S. Cochran, M. Hill, Ultrasound assisted particle and cell manipulation on-chip, Adv. Drug Deliver Rev. 65 (2013) 1600-1610.

[2] M.A. Faridi, H. Ramachandraiah, I. Iranmanesh, D. Grishenkov, M. Wiklund, A. Russom, Microbubble activated acoustic cell sorting, Biomed. Microdevices 19 (2017) 23-29.

[3] A. Sutton, T. Shirman, J.V.I. Timonen, G.T. England, P. Kim, M. Kolle, T. Ferrante, L.D. Zarzar, E. Strong, J. Aizenberg, Photothermally triggered actuation of hybrid materials as a new platform for in vitro cell manipulation, Nat. Commun. 8 (2017) 14700.

[4] X.L. Dong, J. Cai, Z. Wang, Manipulation of living cells by optically induced dielectrophoresis, Manufacturing and Measurement on the Nanoscale, 5th International Conference on Manipulation, 3M-NANO 2015 - Conference Proceedings 7425517, 259-262.

[5] K. Park, S. Kabiri, S. Sonkusale, Dielectrophoretic lab-on-CMOS platform for trapping and manipulation of cells, Biomed. Microdevices 18 (2016) 1-11.

[6] F. Gertz, A. Khitun, Biological cell manipulation by magnetic nanoparticles, AIP Advances 6 (2016) 025308.

[7] G. Lucarini, V. Iacovacci, L. Ricotti, N. Comisso, P. Dario, A. Menciassi, Magnetically driven microrobotic system for cancer cell manipulation, Proceedings of the Annual International Conference of the IEEE Engineering in Medicine and Biology Society, EMBS, 2015.

[8] R. Dreyfus, J. Baudry, M.L. Roper, M. Fermigier, H.A. Stone, J. Bibbette, Microscopic artificial swimmers, Nature 437 (2005) 862-865.

[9] W.F. Paxton, K.C. Kistler, C.C. Olmeda, A. Sen, S.K. St. Angelo, Y. Cao, T.E. Mallouk, P.E. Lammert, V.H. Crespi, Catalytic nanomotors: autonomous movement of stiped nanorods, J. Am. Chem. Soc. 126 (2004) 13424. 
[10] J.E. Perez, M.F. Contreras, E. Vilanova, L.P. Felix, M.B. Margineanu, G. Luongo, A.E. Porter, I.E. Dunlop, T. Ravasi, J. Kosel, Cytotoxicity and intracellular dissolution of nickel nanowires, Nanotoxicology 10 (2016) 871-880.

[11] Y.P. Ivanov, A. Alfadhel, M. Alnassar, J.E. Perez, M. Vazquez, A. Chuvilin, J. Kosel, Tunable magnetic nanowires for biomedical and harsh environment applications, Sci. Rep. 6 (2016) 24189.

[12] C. Gispert, A. Serrà, M.E. Alea, M. Rodrigues, E. Gómez, M. Mora, M.Ll. Sagristá, L. Pérez-García, E. Vallés, Electrochemical preparation and characterization of magnetic coreshell nanowires for biomedical applications, Electrochem. Commun. 63 (2016) 18-21.

[13] A. Sharma, Y. Zhu, S.S. Thor, F. Zhou, B. Stadler, A. Hubel, Magnetic barcode nanowires for osteosarcoma cell control, detection and separation, IEEE Trans. Mag. 49 (2013) 453-456.

[14] F. Byrne, A. Prina-Mello, A. Whelan, B.M. Mohamed, A. Davies, Y.K. Gunko, J.M.D. Coey, Y. Volkov, High content analysis of the biocompatibility of nickel nanowires, J. Magn. Magn. Mater. 321 (2009) 1341-1345.

[15] J. Garcia-Torres, E. Gomez, E. Valles, Measurement of the giant magnetoresistance effect in cobalt-silver magnetic nanostructures: Nanowires, J. Phys. Chem. C 116 (2012) 1225012257.

[16] A. Serrà, E. Gomez, I.V. Golosovsky, J. Nogués, E. Valles, Effective ionic-liquid microemulsion based electrodeposition of mesoporous Co-Pt films for methanol oxidation catalysis in alkaline media, J. Mater. Chem C 4 (2016) 7805-7814.

[17] M. Osumi, Visualization of yeast cells by electron microscopy, J. Electron Microsc. 61 (2012) 343-365. 


\section{Figure 1}

Click here to download high resolution image

(a)

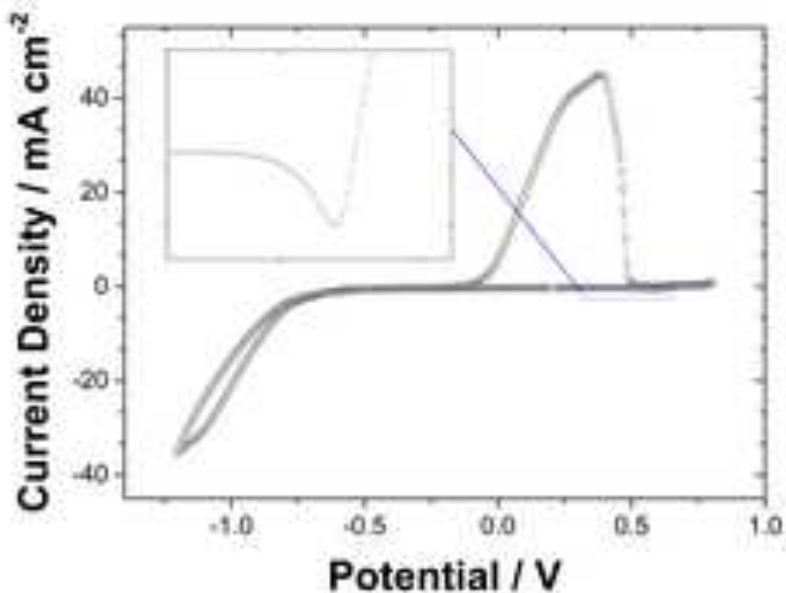

(c)

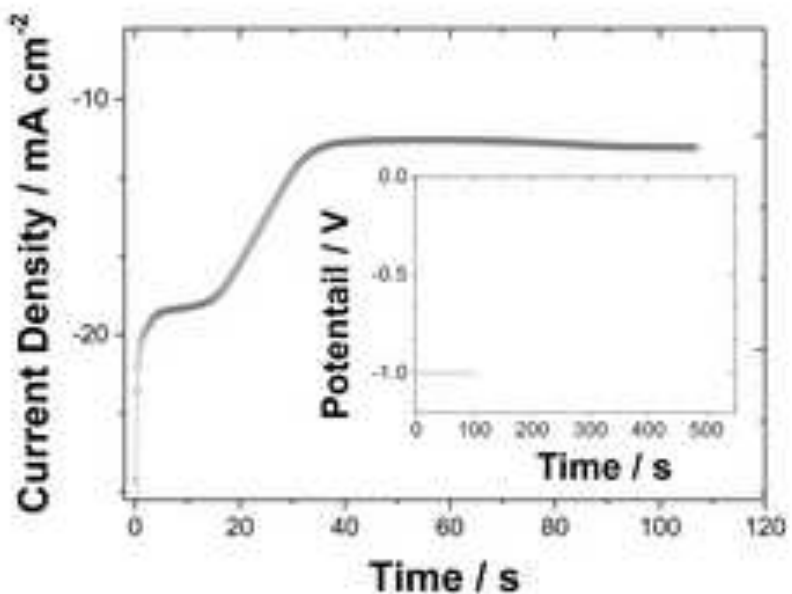

(e)
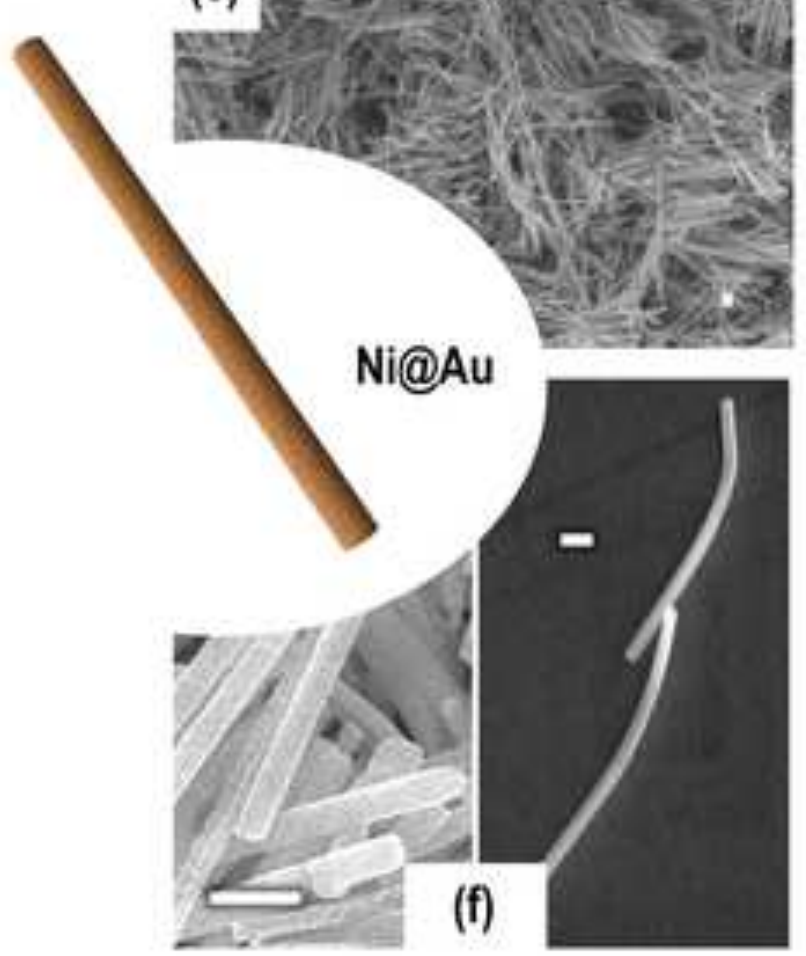

(b)
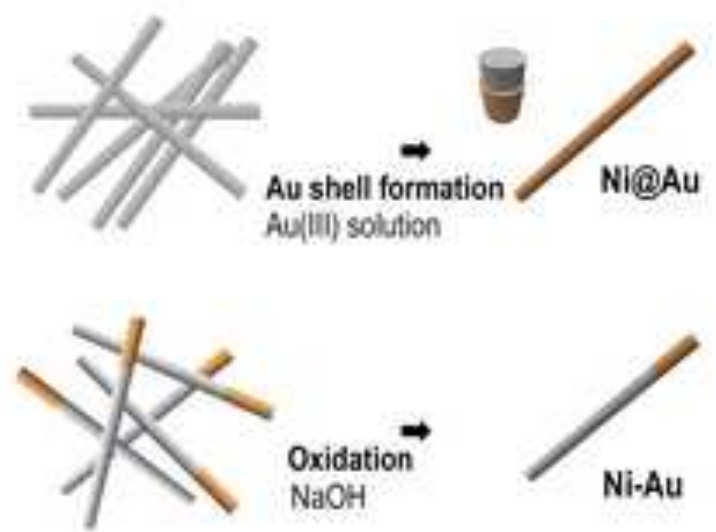

(d)
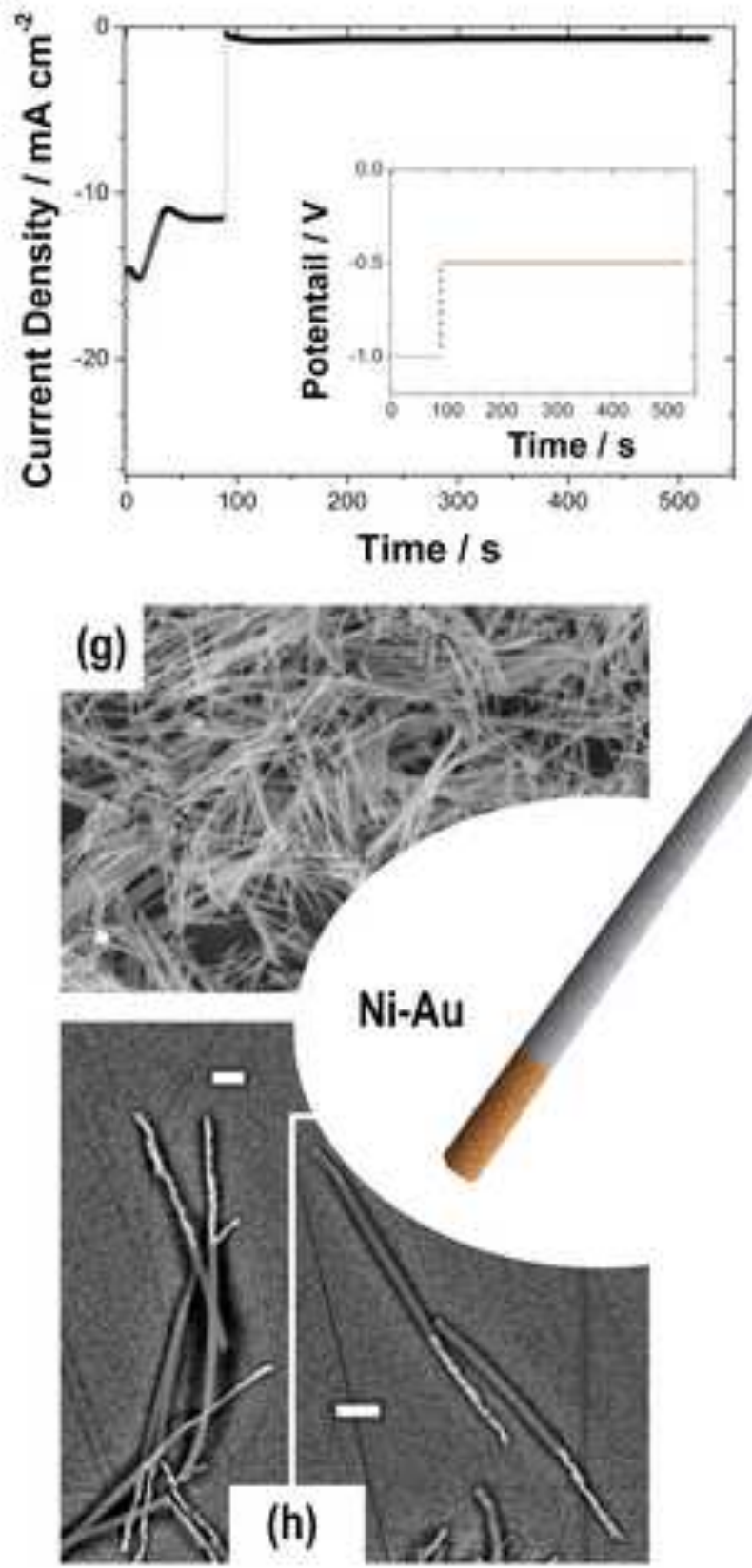


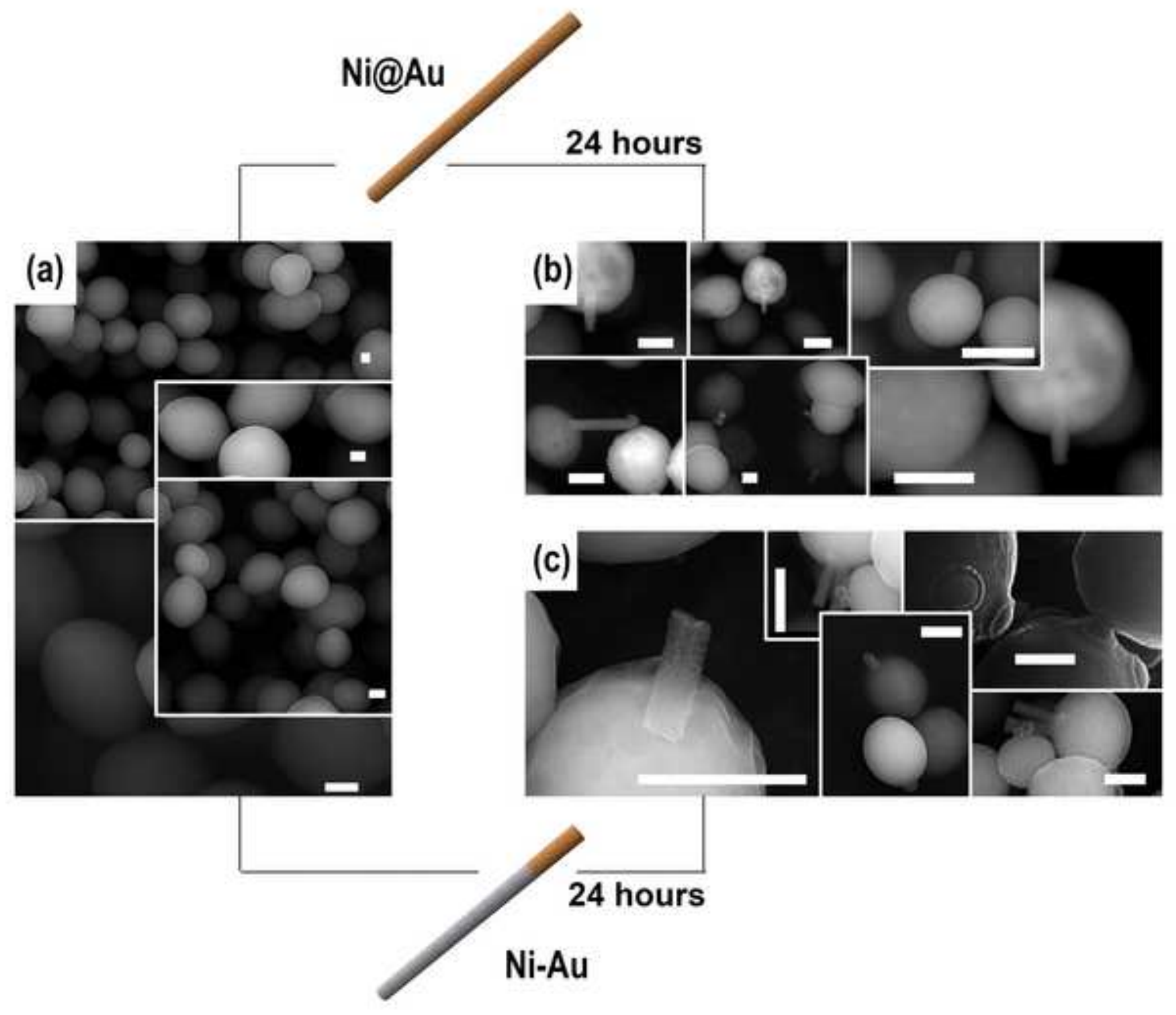


(a)
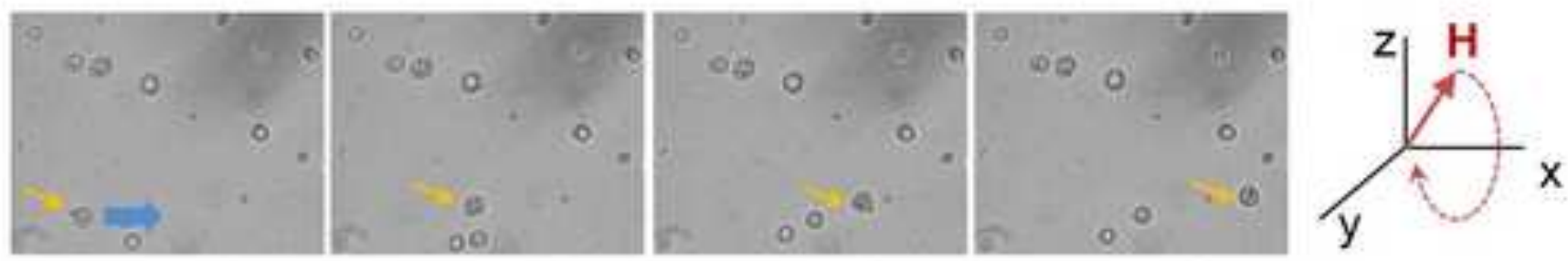

(b)
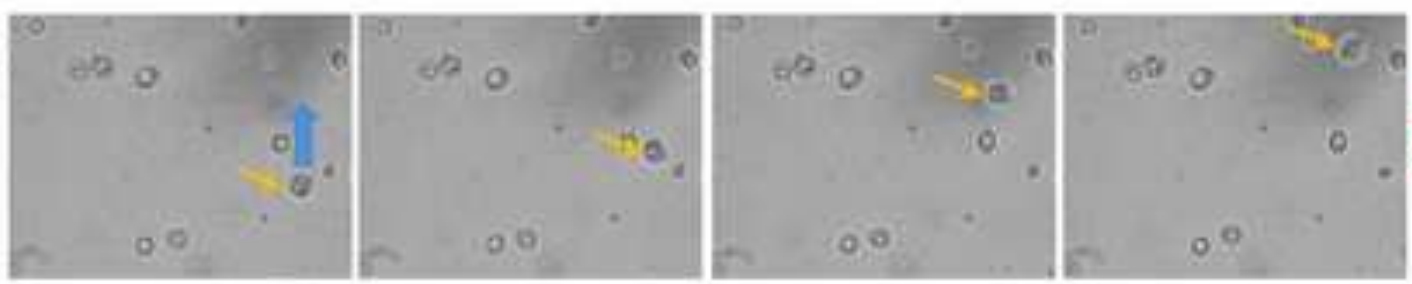

(c)
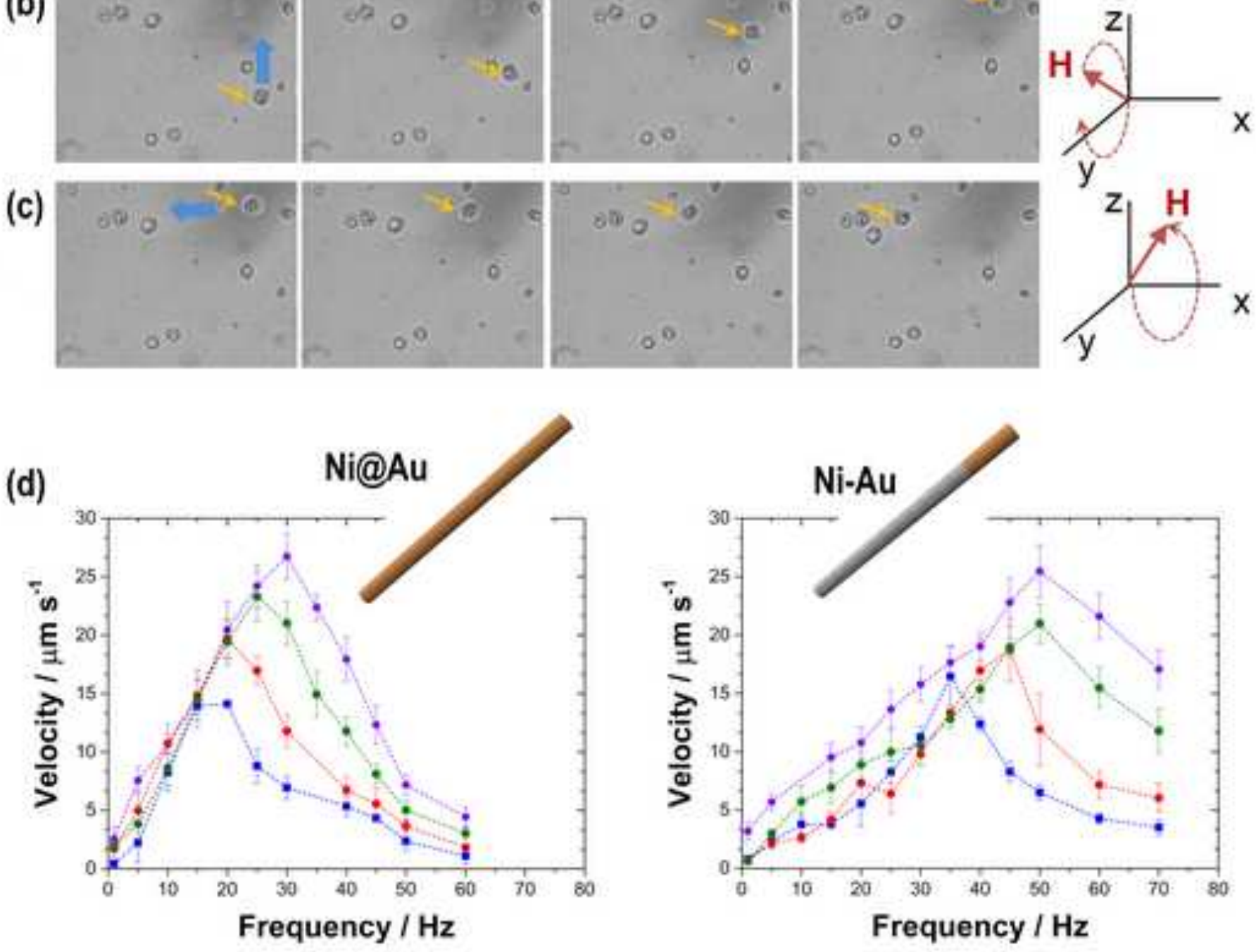

$.5 .4 \mathrm{mT}=4.5 \mathrm{mT} \quad \cdot 3.4 \mathrm{mT}=2.5 \mathrm{mT}$ 
Click here to download Video: Video_S1.mp4 Click here to download Video: Video_si.mp4

4

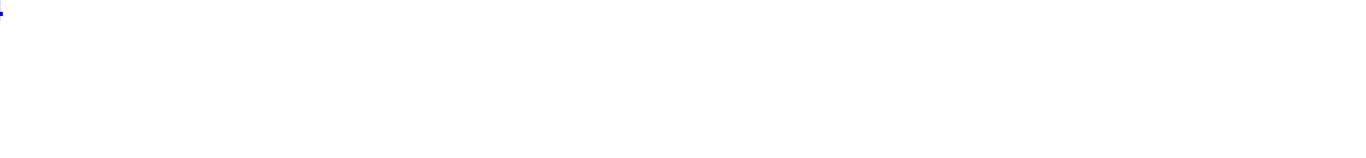

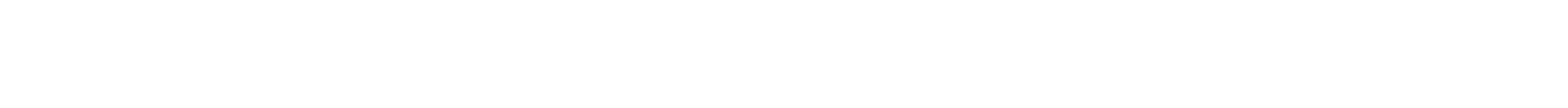

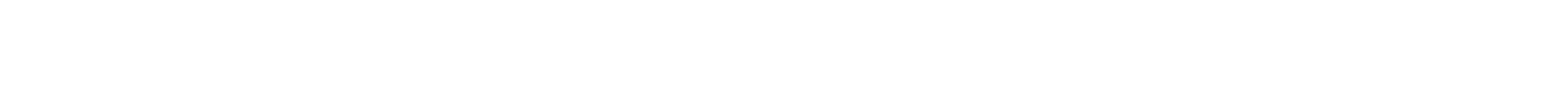

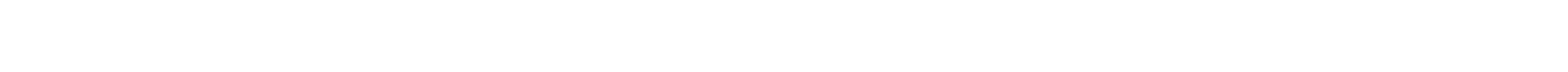
. 政 . .

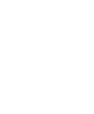



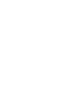

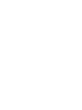

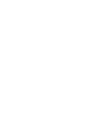





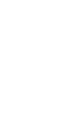

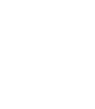

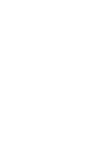

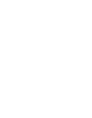

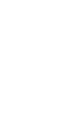

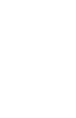
. .

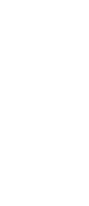
(1) . .

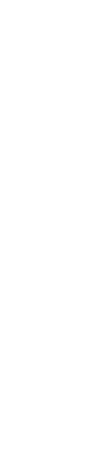


Video_Supporting Information
Click here to download Video: Supporting Information.docx .

Click here to download Video: Supporting Information.docx

(n)

$\sqrt{2}$

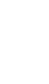

$\sqrt{2}$ (1) (1) $\sqrt{3}$ (1)

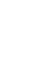
$\sqrt{3}$ (1)

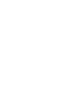

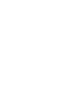
. . .

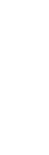

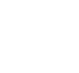

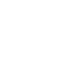
更

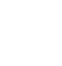

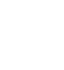

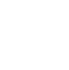
更

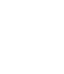

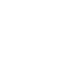

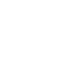

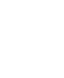

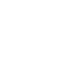

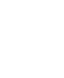

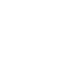

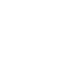

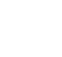

